Commentary

\title{
EU Product Environmental Footprint-Mid-Term Review of the Pilot Phase
}

\author{
Annekatrin Lehmann *, Vanessa Bach * and Matthias Finkbeiner \\ Received: 30 September 2015; Accepted: 13 January 2016; Published: 18 January 2016 \\ Academic Editor: Marc A. Rosen \\ Department of Environmental Engineering, Chair of Sustainable Engineering, Technische Universität Berlin, \\ Strasse des 17. Juni 135, 10623 Berlin, Germany; matthias.finkbeiner@tu-berlin.de \\ * Correspondence: annekatrin.lehmann@tu-berlin.de (A.L.); vanessa.bach@tu-berlin.de (V.B.); \\ Tel.: +49-30-314-79501 (A.L.); +49-30-314-27941 (V.B.); Fax: +49-30-314-21720 (A.L. \& V.B.)
}

\begin{abstract}
The ongoing pilot phase of the European Product Environmental Footprint (PEF) tests the PEF method and develops product category-specific rules (PEFCRs) for selected product categories. The goal of PEF is to address all relevant environmental impacts and the full life cycle of products is acknowledged. However, PEF faces several methodological and practical challenges. This paper presents key findings of a comprehensive analysis of the current status of the PEF pilot phase (mainly based on the evaluation of all draft PEFCRs). Remaining key challenges are: (1) the still open goal and policy outcome of the PEF process; (2) the difficult applicability and, thus, the unclear tangible added value of some PEF rules compared to current life cycle assessment (LCA) practice; (3) the insufficient maturity level of some predefined impact assessment methods and missing reliable methods for prioritizing impact categories; and (4) the fact that, in the worst case, the developed PEFCRs may not support a fair comparability of products. This "mid-term review" of the PEF pilot phase shows that the PEF method and the PEFCRs need to be further improved and refined for a successful policy implementation of PEF, but also for avoiding that unsolved issues of PEF affect the LCA method as such.
\end{abstract}

Keywords: Product Environmental Footprint; PEF; pilot phase; LCA; ISO 14044; comparability; policy

\section{Introduction \& Background}

For two years now the European Product Environmental Footprint (PEF) method has been tested in the pilot phase and - even longer-widely discussed amongst different stakeholders in Europe (and beyond). The so-called PEF method was published in 2013 by the European Commission (EC) [1] as part of the Communication "Building the Single Market for Green Products" [2]. The PEF was published together with the Organizational Environmental Footprint, but the focus of this paper is PEF only. PEF aims at increasing comparability between products by predefining requirements for certain methodological aspects, thus decreasing the flexibility provided by ISO 14044 [3]. ISO represents the worldwide accepted standard on life cycle assessment (LCA). For certain applications, e.g., for using LCA for environmental product declarations, more specifications are required as currently provided in the product category rules (PCRs) defined based on ISO 14025 [4]. As establishing product declarations is one goal of PEF, specifications are needed as well. Thus, in addition to testing the PEF method in practice, the ongoing three year PEF pilot develops product category specific rules (PEFCRs) for selected product categories. PEFCRs are life cycle based rules which complement the general PEF method by providing further specification at the level of a specific product category. They are basically the PEF version of PCRs [5].

The first wave of the pilot phase started in November 2013, and the second wave with focus on feed and food in June 2014. Currently, in total, 24 product categories are covered for which 
draft PEFCRs and screening study reports are developed and published [6]. These documents are commented and discussed by stakeholders, the Technical Advisory Board (TAB), and the Steering Committee (SC). The SC consists of one representative from each EU member state and one from each pilot project, members of the European Commission (EC), and some others (e.g., from the European Environmental Bureau, EEB). The SC is mainly responsible for monitoring the PEFCR development process, for discussing challenges within the pilot phase and for approving the draft and final PEFCRs. The $\mathrm{TAB}$ consists of a group of experts to support the SC by providing technical advice related to the ongoing pilot projects as well as to overall issues related to life cycle assessment (LCA) and environmental analysis; the TAB members are appointed by the SC members (one per member) [7]. The final PEFCRs are expected to be available by the end of 2016 [8].

We have followed the PEF process since the publication of the first draft of the PEF method in 2011. We clearly support the PEF approach to address all relevant environmental impacts and the full life cycle of products, as well as the proposal of further guidance for a harmonized method for quantifying and communicating environmental performance from cradle-to-grave. However, the PEF method and the PEFCR development face several methodological and practical challenges. Some of these challenges were already highlighted in the expert consultation on the first draft of the PEF method-two years before the actual start of the PEF pilot phase-and many of them are now reconfirmed in the pilot phase.

By now, PEF and its opportunities and challenges are subject of several publications and position papers from various stakeholders including academia $[9,10]$, industry [11-13], as well as policy-makers [14]. The challenges regarding PEF refer mainly to the missing specified goal and intended policy application of the PEF process, the "comparability over flexibility" respectively "one-size-fits-all" approach [12], and the lack of conformity with international standards.

Here, it should be noted that the intensive work of the pilot projects is acknowledged and that, by now, PEF helped to spread the LCA concept amongst industries. However, with regard to the aspects covered in the draft PEFCRs presented as of now, several challenges and concerns raised in the existing publications are reconfirmed.

It also should be noted here, that the EC has taken many efforts to tackle the methodological and practical challenges of the PEF pilot phase. For example, workshops are organized and cross-cutting working groups are established to discuss issues like impact assessment, weighting, or the end-of-life (EoL)-recycling formula, and to try to find solutions/consensus. Moreover, continuously updated and specified guidance for the pilot phase is provided.

Topics like weighting, suitable impact assessment method, or prioritization of impact categories are well-known challenges in current LCA practice and many LCA practitioners would like to see further discussions here. Thus, on the one hand, we acknowledge that PEF picks up these topics. On the other hand, resources spent in the pilot phase are further increasing, but the learnings (e.g., with regard to prioritization of impact categories) and improvements are not significant.

The goal of this paper is to present the key findings of a comprehensive analysis of the current status of the ongoing PEF pilot phase. The pilot phase is now already in its second half and as such, a review of the achievements so far is feasible. The remaining challenges are described and recommendations for improvement, including the ones provided/recognized by the EC are presented. It will be shown, that an improvement of the PEF method itself and of the PEFCRs is strongly needed, if the many resources, which were already spent to develop the PEFCRs, are supposed to lead to a successful and relevant policy implementation of the PEF method.

\section{Methods}

A comprehensive analysis of the PEF pilot phase was conducted considering previous work of the authors and other existing PEF related publications $[9,10,12,15,16]$. In addition, all available documents developed within the pilot phase were evaluated. This includes the 24 draft PEFCRs (with up to 100 pages) and the 24 screening study reports (with up to 300 pages) prepared by the individual pilot 
projects, as wells as more than 10 issue papers and other documents provided by the EC addressing various relevant topics like prioritization of impact categories, benchmarking, etc. In addition to this the authors have been actively participating in the PEF pilot phase on different levels (as stakeholders in some pilot projects and as TAB members) and, thus, gained direct insight into the pilot phase. Based on this analysis the key challenges of the pilot phase were identified.

The results of this comprehensive analysis of the pilot phase including recommendations for improvement are presented in the following section complementing the findings from previous publications.

\section{Results and Discussion}

Several methodological and practical challenges were identified in the PEF pilot phase. Basically, all these specific challenges can be related to four main questions:

1. What is the specific goal and expected policy outcome of the PEF process?

2. Are the PEF rules applicable? Do they provide an added value compared to current LCA-practice?

3. Are the predefined impact categories and related life cycle impact assessment (LCIA) methods suitable for comparisons and comparative assertions and is the procedure for prioritizing impact categories (e.g., for communication) reasonable?

4. Will the PEF rules support comparability or rather reproducibility?

The specific challenges identified are described in the following four subsections, allocated to one of the four questions above. Sometimes the specific challenges can be related to more than one question (e.g., EoL recycling formula), thus, to more than one subsection. In these cases we chose the subsection we found predominant. Specific challenges, which were already discussed in previous publications (e.g., choice of the LCIA method) are only shortly addressed in the respective subsection, but not discussed in detail.

An overview of the specific challenges and their relation to the four questions is given in Figure 1 together with a timeline, which indicates the approximate dates when the challenges were reconfirmed within the pilot phase, i.e., when deeper discussions on these topics started.

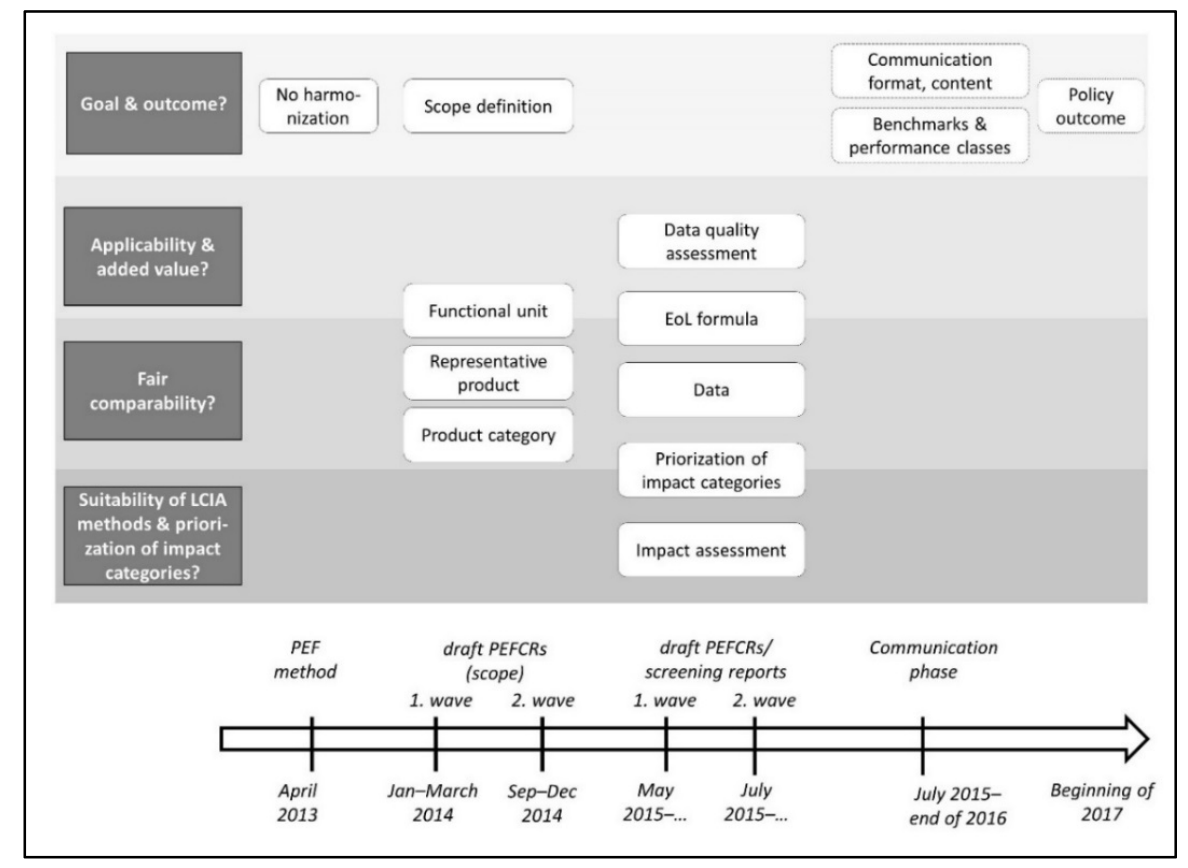

Figure 1. Overview on key challenges of the PEF pilot phase and approximate dates when the related discussions started (December 2015). 


\subsection{Goal and Outcome of the PEF Process}

PEF is a method which is supposed to allow for a multi-criteria analysis of the environmental performance of products and which intends to harmonize existing standards. Harmonizing standards is an appreciated goal, but so far we do not see that this is achieved with PEF [9]. Rather, another standard is added to the already existing ones. Moreover, the current scope definition in the draft PEFCRs is nonspecific and flexible regarding the exact aims and the intended application of the PEFCRs as well as regarding the intended format and content of the communication [10]. Thus, the goal and expected outcome of the PEF process is still unclear. The EC stresses that these key aspects can be specified only after the pilot phase is completed, based on the experience and knowledge gained during these three-year testing phases. Thus, the EC keeps this question deliberately open. However, in practice, it is well known that, especially, the aim and intended application of the PEFCRs need to be clearly defined before specific rules that are fit for purpose can be established. A clear and as simple as possible policy implementation scenario, which is agreed with those policy-makers responsible for the implementation, is much more relevant for practical implementation than, for example, trying to find an EoL recycling formula.

\subsubsection{Deliberately Left Open PEFCR Scope}

According to the Guidance for the implementation of the PEF method during the PEF pilot phase several goals and application are possible for the developed PEFCRs. This includes, amongst others, in-house product improvement or business-to business (B2B) or business-to-consumer (B2C) green claims, with or without comparisons or comparative assertions of products within one product category [8]. Whereas in current LCA practice the scope is determined based on the goal of a study and its application, the scope in the current draft PEFCRs is defined without being explicit in the intended application. To determine the goal of the PEFCRs only after the PEF pilot phase has ended is a deliberately-chosen approach by the EC. The pilot phase is seen as a testing phase to specify the PEF goal and method based on gained experience/knowledge. However, inconsistencies amongst the PEFCRs result from the missing goal, which bares a significant risk regarding the applicability and meaningfulness of the final PEFCRs. For example, currently, some of the pilot projects introduced specified rules for subcategories (e.g., graphic, packaging and tissue paper in the intermediate paper pilot), while others (e.g., feed) do not. For the feed pilot project, this could mean that one benchmark is established for this broad product category. Hence, the environmental performance of feed for different animals (e.g., pigs and cows) could be compared, while in practice, from the viewpoint of a farmer, it would be more reasonable to compare different feeds for one animal (e.g., pigs or cows). Moreover, as the composition of feed varies for different animals, specific rules for modelling the product system may be required, which are currently not provided. Hence, the current PEFCR draft should be for in-house improvements, rather than for comparisons or comparative assertions for B2C. In the worst case, the PEFCRs developed may neither be suitable for supporting comparisons or comparative assertions nor for in-house product improvements. To avoid this outcome the PEFCRs should be double-checked and may need to be adjusted once their goal is clearly defined. To do so is actually planned by the EC. However, so far this update will take place probably only three years after the pilot phase (three years is the validity time for all established PEFCRs). This could mean, that these PEFCRs are used for decision-making even though they are not up to date and might show weaknesses.

\subsubsection{Deliberately Left Open Communication Format}

As a consequence of the unclear aim and intended application of the PEFCRs it is also yet unclear which communication vehicle-if any-will be chosen. During the pilot phase each pilot project should test different communication vehicles for different potential applications, e.g., product labels for B2C communication or environmental reports for B2B communication $[8,17]$. Especially regarding the development of a potential B2C-label, benchmarks and environmental performance classes deserve 
special attention as they may be the basis to communicate the environmental performance of a product [8]. A rough guidance on how to determine benchmarks and environmental performance classes is provided in an EC issue paper [18], but the actual procedure is up to the individual pilot project. However, as the definition of benchmarks and performance classes is not a simple and straightforward task, a more detailed guidance for the pilot projects is needed. Maybe this missing clear guidance is one reason why so far only few pilot projects presented ideas and approaches for defining environmental performance classes. The few proposed approaches are mainly based on scenarios for worst and best case regions for electricity. However, environmental performance classes defined based on regional electricity mixes can lead to unfair market conditions and provide wrong incentives (see Section 3.4.2.). A comprehensive approach for determining benchmarks and performance classes is presented in Gül et al. [19]. The authors transparently show the efforts and challenges to define benchmarks and performance classes as well as their limitations with regard to using them as basis for comparisons and comparative assertions.

In addition to this, it is unclear if and how information listed under the PEFCR's additional environmental information will be considered in the communication of the environmental performance of products. Additional environmental information may refer to environmental impacts that cannot yet be properly assessed within LCA (e.g., biodiversity) or to materials that are not included in the product system of the representative product (e.g., organic materials), but which are relevant for determining and communicating the environmental performance of a product. For B2C communication such relevant information or specific criteria need to be included as done in existing environmental declaration systems, like the EU ecolabel [20].

The topic of future communication was one key topic in the midterm conference on the environmental footprint pilot phase, which took place from 3-4 November 2015. However, clear statements from the EC regarding the intended application or communication, for example if a label should be established or not, are still missing. If there will be any form of a PEF-label at all, it is-due to its voluntary nature- just another label added to the many labels we already have and in that sense may not reduce, but increase, the proliferation of labels.

\subsection{Applicability of the PEF Rules and Their Added Value}

Some methodological key aspects or rules of the PEF method come along with a high-especially bureaucratic-effort. At the same time the added value of these rules is not clearly visible. The rules for defining the functional unit as well as the predefined EoL recycling formula are two examples and are described below. Another example for a PEF rule with little tangible added value but high effort is the scheme for data quality assessment (see also [9]).

\subsubsection{Definition of the Functional Unit}

The detailed requirements of the PEF method for defining the functional unit ("what", "how well", "how much", "how long") are rather theoretical as several pilots fail to address them properly. For example, the requirement "how long" is often related to the consumption of the product (e.g., one drink), whereas it should rather refer to the product lifespan (e.g., four weeks). Furthermore, the functional units proposed are often not suitable for meaningful comparisons and comparative assertions as they neglect relevant quality aspects, i.e., do not properly define "how well". For example, instead of the quality of the product often less relevant surrounding factors, which are not important for the environmental performance of the product (e.g., serving temperature of drinks), are considered. Consequently, a tangible added value of the PEF requirements for defining the functional unit compared to the current practice of LCA is not visible.

\subsubsection{EoL Recycling Formula}

The predefined EoL recycling formula can lead to inadequate results. For example, how often a material can be recycled is not considered in the formula. Hence, a material that is recycled once gets 
the same burden/credit as a material that is recycled several times. In addition, the formula favors the incineration of materials instead of their reuse and recycling: whereas the benefits of reuse and recycling of materials are only credited with $50 \%$, the incineration benefit is $100 \%$. Thus, the use of one way packaging like plastic bottles would have a better environmental performance because they are incinerated at the EoL whereas returnable packaging like glass bottles, which often are recycled several times, would perform worse. Consequently, the formula may lead to (1) questionable results; which will not lead to the reduction of environmental impacts and (2) results which contradict the European Directive on waste 2008/98/EC, which clearly states that reuse and recycling are more favorable than energy recovery [21]. The difficulties of interpreting the results were already acknowledged within the pilot phase and intensively discussed, for example in a specific EoL workshop. However, sufficient solutions were not yet found. As a first step the EC encourages the pilot projects to test other EoL recycling formulas next to the PEF formula and supports this by providing a spreadsheet which also includes the formulas for the 0/100 (recycled content) and 100/0 (cut off) approaches. However, so far only few pilots actually apply other EoL formulas. Thus, a comprehensive comparison of available formulas is not possible. Additionally, one of the main challenges in modeling the EoL phase-allocating benefits for reuse and recycling of materials-is not solved within the alternative formulas provided.

\subsection{Impact Assessment and Prioritization of Impact Categories}

None of the ISO standards related to LCA predefine impact categories and LCIA methods to be used in LCA studies. However, some existing PCRs make more clear recommendations, which impact categories to consider and sometimes which method should be used. Thus, in current LCA practice the considered impact categories as well as the used methods differ depending on the study and PCR. Following the "comparability over flexibility approach", PEF provides a predefined set of 14 impact categories to be assessed with 15 predefined LCIA methods [1]. During the pilot phase it became apparent that aspects of marine eutrophication have to be taken into account as well. Thus, an additional category was added [8]. For the potential communication of the environmental performance of products, especially for B2C communication, it is foreseen to focus on relevant impact categories only. Challenges are related to the scientific robustness and applicability of the predefined LCIA methods as well as to the procedure for prioritizing impact categories.

\subsubsection{Impact Assessment Methods}

Several of the (now 15) recommended LCIA methods are classified as not being very mature $[10,22]$. This includes the ecological scarcity method for assessing water depletion and the model for assessing abiotic resource depletion of minerals (ADP indicator) based on an economic reserve base. The insufficient maturity level of some LCIA methods is also recognized by many pilot projects and the EC. Thus, the EC organized a workshop to discuss the suitability of the USETox method for communication. Currently, the Joint Research Centre (JRC) supports the USEtox developer in a comprehensive update of the background data. Hence, an updated version might be available in the future. Moreover, the EC encourages the pilot projects to test alternative LCIA methods, but only few pilots indeed did/do so. The EC already announced that some LCIA methods, e.g., land use, water use, resources, or particulate matter will have revised recommendations probably by the end of 2016. Though this is acknowledged, we do not really understand why non-robust LCIA methods cannot be exchanged by better-existing ones already now (e.g., water and resource depletion)_adding an additional impact category during the ongoing pilot phase was obviously possible. More suitable LCIA methods for water depletion are the methods of Pfister et al. [23] and Berger et al. [24] and for resource depletion the method of Schneider et al. [25,26], which have been available since 2009, thus before the PEF method was published. Furthermore, updating the impact assessment methods only after all 24 PEFCRs are finalized should automatically lead to an invalidity of these PEFCRs as the determined relevant impact categories might not be valid anymore. 


\subsubsection{Prioritization of Impact Categories for Communication}

Currently, no commonly accepted method for prioritizing impact categories exists, but several LCA practitioners may appreciate more guidance on this topic. According to the PEF method, normalization and equal weighting were foreseen to be used in the screening studies to identify the most relevant impact categories, i.e., to prioritize the impact categories for the product system considered $[1,8]$. However, the approach to use normalization and equal weighting for identification of relevance has been questioned in LCA practice since many years and now also by some pilot projects. There are several reasons why normalization and equal weighting are not appropriate to determine relevant categories. The underlying concept of normalization is to set the emissions of the product system into relation with the emissions of a reference region (e.g., World or Europe). If the emissions of a region are small (e.g., within Europe) the normalization factor is small as well and the corresponding normalized result is rather high. Contrarily, the higher the emissions in one region are, the smaller is the normalized result. Thus, the relevance of the impact category decreases for example when global emissions are taken into account. This is the reason why, next to normalization, weighting also has to be applied to determine the relevance of impact categories. However, using equal weighting as suggested by PEF basically leads to the fact that the normalized results determine the importance of the impact categories. Establishing a specified weighting method agreed on by several stakeholders is a very ambiguous goal, which could not be achieved so far [27]. Thus, it can be assumed that, also in the future, impact categories will be prioritized based on normalization and equal weighting. However, the argument that the specific amount of emissions in a product system is more important when the overall emissions in the reference region are low is very questionable (which is the case when normalization and equal weighting is applied) as the reversed argument could be valid as well: the emissions of a product system are considered worse, when the emissions within a region are already very high.

For the exemplary impact category acidification (see Figure 2) acidifying emissions within Europe are relatively low as several national and European laws and regulations exist since the 1980s to reduce these emissions significantly. In other parts of the world e.g., China, where many of the products sold in the European market are produced, such regulations often do not exist or are not implemented adequately. Thus, the amount of acidifying emissions is significant. Using normalization and equal weighting to determine the relevance of impact categories would identify acidification as a relevant category, when Europe is considered, but as less relevant when global production values are taken into account. This may not reflect reality properly.

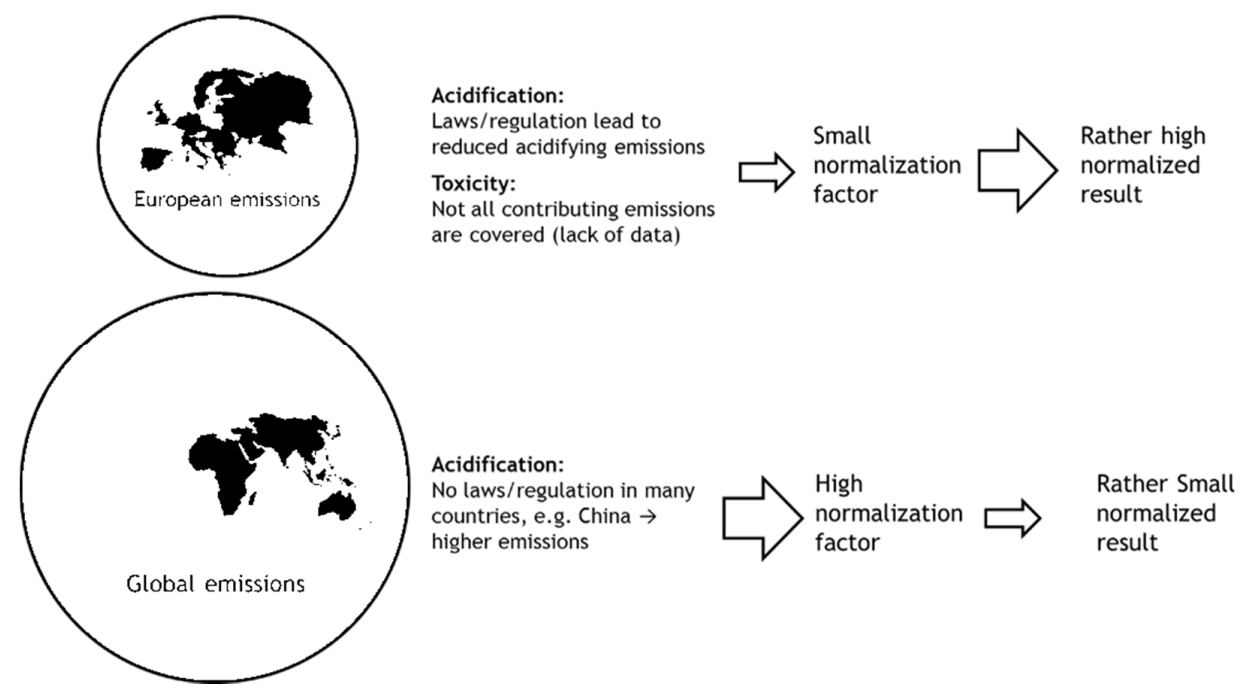

Figure 2. Illustrative example on challenges of normalization and equal weighting for prioritizing impact categories. 
Furthermore, data for the calculation of normalization factors are often not available. For example, for the toxicity categories tens of thousands of substances have to be considered, but data for far less than 1000 actually exist. Moreover, the normalization factors provided by the EC are only based on European, not on global, data, which can distort results because emissions in the product system occur not only in Europe, but all over the world (see Figure 2). The EC initially intended to provide global data, but due to data availability this could not be accomplished.

In the meantime, based on the outcome of the screening studies (in the pilot projects the impact categories freshwater ecotoxicity, human toxicity and water depletion were predominantly identified as relevant), mainly due to the facts described above. The challenges related to normalization and equal weighting are also recognized by the EC. Thus the EC decided that normalization and equal weighting should not be used as a sole basis to define which categories are relevant, but that justified expert judgement is additionally needed [8]. Now each pilot has to define, individually, which categories are relevant, thus which ones should be considered for communication. As a consequence, often impact categories for which robust impact assessment methods are not yet available (e.g., for resource depletion and toxicity) are excluded per se. We agree that non-robust results should not be communicated, but this discussion simply confirms the challenges related to communication and comparisons. Excluding certain categories could lead to a burden shifting, i.e., when the impact categories, which are communicated, are improved at the expense of other categories, which are not communicated, but still may be relevant for the product category. Furthermore, PEF requires to select minimum three impact categories for communication [8]. Thus, eventually only three categories may be chosen, which would be less than in current LCA practice, where at least the "classical 5" (climate change, acidification, eutrophication, photochemical ozone formation, and ozone layer depletion) are evaluated.

Even though the JRC is working on improved normalization factors, which will be available probably by 2017, our concerns related to using normalization to prioritize impact categories will not be solved by improved normalization factors.

\subsection{Achieving Comparability}

PEF proposes the approach of "comparability over flexibility". Comparability means that alternatives, e.g., products within one product category, can be compared based on fair and robust rules. Reproducibility on the other hand means that, if everybody applies the same defined rules, the same results will be obtained. This distinction is important as comparability and reproducibility are two different things, which often exclude each other. To enable fair comparisons, rules for conducting LCAs need to be defined and consensus on methodological key aspects needs to be found (e.g., definition of the representative product, use of primary and secondary data, allocation rules). This is done in the PEF pilot phase. PEFCRs are defined based on a consensus reached within the TS, which consists of stakeholders from industry, academia, NGOs, etc. Moreover, other stakeholders can comment of PEFCR drafts in public consultations. This is basically in line with the current practice of PCR development. In addition, working groups (e.g., cattle model working group) are established consisting of members from different TSs / pilot projects (e.g., dairy products, leather, meat) to discuss cross-cutting issues and to find consensus, e.g., on allocation rules. However, several challenges remain. First, consensus cannot always be achieved (e.g., in the cattle model working group). Second, the predefined rules may rather support reproducibility instead of comparability. Hence, PEF rather follows an approach of "reproducibility over flexibility" instead of "comparability over flexibility" and as a result PEF would not support a fair competition of products. The following examples on the definition of the representative product for a product category and the definition of data sources highlight the challenge of PEF to support a fair comparability of products. 


\subsubsection{Definition of the Product Category and the Representative Product}

Challenges related to the definition of the product category are already addressed in Section 3.1.1 related to the discussion of the PEFCR scopes and in Lehmann et al. [10]. The concept of the representative product(s) is a new one established by the EC within the PEF method. The basic idea is to determine one product which is representative for the entire product category. For each representative product a PEF screening study is carried out, which provides insight into the relevant life cycle stages, processes, and impact categories of the product category. The representative product is also the basis for the proposed approach of establishing benchmarks and environmental performance classes for the product category. To identify significant potential environmental impacts the representative product should include all processes that could have environmental impacts. For defining the representative product, the EC proposed the approach of market share. However, if the representative product is defined based on market shares, these impacts are not necessarily reflected, because the market average is not necessarily the "environmental" average. Thus, higher quality products with a low market share may be discriminated against lower quality products. Moreover, certain assumptions in the bill of materials (e.g., considering additives by their maximum dose allowed or neglecting certain materials) can easily lead to over- or underestimations of environmental impacts of the representative product and consequently to the fact that real products always will perform better (or worse) than the defined representative product.

According to the guidance for the implementation of the PEF method during the PEF pilot phase [8] the supporting studies which are conducted for real products (contrary to the screening studies performed for the representative product, which can be a virtual one) are used to refine the benchmark and the environmental performance classes. This is a promising approach in theory, but only adequate if sufficient supporting studies are conducted. In fact, the three supporting studies per pilot project proposed by the EC are probably not sufficient for a real fine-tuning, especially not when the pilot project introduced subcategories: as each subcategory has one representative product, it would mean that a pilot with e.g., three subcategories would need to conduct at least nine supporting studies for real products, but due to restricted resources within the pilot projects this seems to be very unlikely. In addition, a reality check of the applicability and meaningfulness of the PEFCRs would require supporting studies conducted by independent organizations. However, the supporting studies are often performed by companies represented in the pilot projects developing the PEFCRs and the studies are often supported by the same consultants drafting the PEFCRs, i.e., it is the ones that drafted the rules who also test them. Thus, in order to establish reliable benchmarks and performance classes more and independent supporting studies are needed. However, for doing so many more resources would be needed.

\subsubsection{Data-Generic vs. Specific}

The EC provides guidance on when to use generic and specific data. The intention is to drive the provision of high quality data for relevant processes and to provide generic data when specific data are not available [8]. Most of the pilot projects use generic data for modelling the background system as specific data are currently often not available. This is in line with current LCA practice when in-house studies are carried out, but bares risks, with regard to a potential future use of PEF for comparisons and comparative assertions. If the data source is fixed, thus if this data is used for all products within one product category, the reproducibility of the PEF studies is increased, while a fair comparability of these products is not supported. For example, in some food and feed pilot projects, background processes, which were identified as being relevant (e.g., agricultural production), are modeled with generic data. However, the farming technique (e.g., intensive vs. organic farming) can have significant influence on the overall environmental impacts. Consequently, potential benefits of "greener" materials are not taken into account. Thus, "greener" products may be punished while "bad" products may be rewarded. A potential future PEF-label based on such a modeling approach 
would contradict existing European labeling standards e.g., the Eco leaf, which aims at supporting organic farming [28].

The challenge of data availability is also recognized within the PEF pilot phase. With regard to generic data, PEF requires using publicly available data from databases like the European life cycle database (ELCD)). However, due to limited data availability pilot projects currently often also used data from private data providers, e.g., Ecoinvent or Thinkstep, thus data which needs to be paid for.As a result a "call for data" was launched (first call of data was out in January 2016), i.e., data providers are asked to provide generic data, which will improve the data availability for future PEF studies However, the challenge of the availability of specific data is not yet solved. Even though some of the involved companies provide specific data, specific data for many background systems especially outside of Europe will still be lacking. Having this in mind, we recommend that the use of primary/specific data will be strengthened in the PEFCR (at least in future ones) to avoid that the PEFCRs rather increase reproducibility instead of comparability.

Moreover, in several pilot studies energy intense processes are analyzed by applying country-specific electricity mixes, because it is believed to reflect the real impacts of a particular product as closely as possible. However, when comparative assertions of products is the goal of the PEF, this approach means that products produced (and/or used) in countries with coal-based electricity mixes, for example, will always perform worse in the impact category climate change than countries using nuclear power. However, the overall energy policy of the member states of the EU is not really an issue to be solved by PEF. However, small and medium enterprises (SMEs), which cannot move their business easily from e.g., Poland to France will be worse according to PEF even if their own operation, for which they have control, is more energy efficient than the one in France. Thus, using country-based electricity mixes does not reflect actual process improvements and, moreover, would lead to unfair market conditions and unintended consequences. Applying the European electricity mix for all energy intense processes could solve both issues.

Of course, a company A e.g., in Poland, which decides to purchase green electricity and, thus, probably lowers its environmental impacts, compared to another company B in Poland, which uses the electricity mix (mainly based on coal) should be rewarded. However, in current LCA studies-intended for comparisons-benefits from using green electricity (i.e., lower environmental impacts) can only be considered if there is no double-counting. For example, company A buys hydropower from Norway; thus, its electricity consumption is modeled with green electricity. For company B the source of electricity is not known. Thus, the modeling for company B is done using a country-specific electricity mix, which also contains a share of hydropower from Norway (the share bought by company A). As a consequence, both companies A and B reduce their environmental impacts by using partly "the same" hydropower from Norway-the environmental benefits of the green electricity hydropower is double-counted. As this challenge is not yet solved, we recommend using the European electricity mix for comparisons. When PEF is applied for internal product evaluation the company specific electricity mix should be used. Furthermore, if a company produces their own electricity this should be accounted for as well.

\section{Conclusions}

As stated earlier we support the PEF approach to address all relevant impact categories and the full life cycle of products. However, PEF faces several methodological and practical challenges. Some of them were already raised in the last years (since 2011) and were reconfirmed in the pilot phase. In addition, new challenges became visible, as well as the fact that the draft PEFCRs from the different pilot projects suffer from huge inconsistency with regard to e.g., the functional units or data, etc. Overall, the challenges and concerns regarding PEF can be summarized as follows:

- The goal and the expected policy outcome of the PEF process are still unclear! The lack of a clear definition of the aim and intended application of the PEFCRs and, thus, the lack of focus with regard to the defined rules for the product category present a threat: eventually the PEFCRs may 
neither adequately support the comparison of products within one product category nor may they provide a robust basis for internal in-house optimization.

- Some PEF rules are difficult to apply and, moreover, show no visible added value compared to current LCA-practice! Many practical challenges of the PEF method (e.g., application of the EoL recycling formula or definition of the functional unit) were already raised before the start of the pilot phase and are now reconfirmed. Other challenges additionally became visible (e.g., the availability of primary data or publically available generic data). Overall, in its current form the draft PEFCRs do not provide added value to the current practice of LCA and other environmental assessments/communications but rather an added effort.

- Several LCIA methods proposed by the PEF method are not mature and a reliable method for prioritizing impact categories for potential communication is missing! The fact that some LCIA methods are not mature and need to be interpreted cautiously was confirmed in the pilot phase, as well as the fact that normalization and equal weighting is not suitable for determining the relevance of impact categories. Moreover, the selected impact categories for communication do not necessarily reflect the actually relevant environmental impacts of a product (which also affects a fair comparability of products).

- As of today, the developed PEFCRs do not seem to support a fair comparability of products! It was confirmed in the pilot phase that the variability in the real world cannot be easily transferred into fixed modeling approaches or a representative product. Even reproducibility will hardly be achieved, let alone comparability.

By now, many of the challenges addressed in this paper are also recognized by he EC and several actions were taken to tackle them. This includes the development of several issue papers (e.g., for defining benchmarks or on including biodiversity in the additional environmental information), the realization of workshops (e.g., on USETox and the EoL recycling formula), the formation of horizontal working groups working on cross-cutting issues (e.g., energy modeling) or the call for data. Additionally, the EC started to specify the intended application and communication format of the PEF.

Moreover, the EC announced that the experience and findings from the pilot phase may be considered in the updated version of the PEF method after the pilot phase. The potential revision refers for example to reconsidering the requirements for the definition of the functional unit, to specifying how to define product categories, to providing further instructions on how to apply the EoL recycling formula and to providing mature impact categories. These efforts in tackling the challenges are acknowledged by the authors and we hope that the efforts will remain and further increased and that specifications/concrete announcements (e.g., on the intended application) are further specified. For example, as highlighted in this paper, we think that a clear definition of the aim of each pilot project with regard to comparability or comparative assertions is highly required to ensure applicable, meaningful PEFCRs. Moreover, we encourage the EC to replace some of the predefined LCIA methods by more mature ones (e.g., water and resource depletion), to use the European electricity mix for energy intensive processes instead of country-specific mixes in order to avoid unfair market situations and to ensure a proper level of consistency between the pilot projects. We also, as the EC, encourage the pilot projects to further test different alternative EoL recycling formulas and LCIA methods.

Moreover, we hope that the announcements of the EC to consider recognized challenges/ improvements will be in fact implemented. We stress this here, as experience in the last years unfortunately showed that often even obviously factual recommendations provided were not considered in the end. For example, recommendations for some alternative LCIA methods were already provided in 2011 on the first draft of the PEF method, but ignored. Furthermore, we would like to highlight again that some of these challenges were already common knowledge in current LCA practice (e.g., the limitation to use normalization and equal weighting for prioritization). Now these challenges were newly "identified" in the pilot phase and discussed. Though discussions are good, the question arises if the resources spent to come to the conclusions (time, money) were really worth it. 
Acknowledgments: We gratefully acknowledge that parts of the research presented here are prepared on behalf of the German Environmental Agency (Umweltbundesamt) as part of the environmental research plan-project code number 371295 337-and financed with federal funding.

Author Contributions: Annekatrin Lehmann is the leading composer of this manuscript. Vanessa Bach and Matthias Finkbeiner contributed substantially to the structure and text of the manuscript. All authors contributed to the content, i.e., to the evaluation of the PEF-pilot phase. All authors proofread and approved the final manuscript.

Conflicts of Interest: The authors declare no conflict of interest.

\section{References}

1. European Union. Commission Recommendation of 9 April 2013 on the use of common methods to measure and communicate the life cycle environmental performance of products and organisations. Available online: http:/ / eur-lex.europa.eu/legal-content/EN/TXT/?uri=CELEX\%3A32013H0179 (accessed on 6 January 2016).

2. European Commission. Building the Single Market for Green Products. Facilitating Better Information on the Environmental Performance of Products and organisations. Communication from the Commission to the European Parliament and the Council. COM(2013) 196 Final; European Commission: Brussels, Belgium; Luxembourg, Luxembourg, 2013.

3. ISO. 14044 International Standard-Environmental Management_Life Cycle Assessment-Requirements and Guidelines; International Organization for Standardization: Geneva, Switzerland, 2006.

4. ISO. 14025 International Standard-Environmental Labels and Declarations-Type III Environmental Declarations_-Principles and Procedures; ISO: Geneva, Switzerland, 2006.

5. Minkov, N.; Schneider, L.; Lehmann, A.; Finkbeiner, M. Type III Environmental Declaration Programmes and harmonization of product category rules: Status quo and practical challenges. J. Clean. Prod. 2015, 94, 235-246. [CrossRef]

6. European Commission Product Environmental Footprint News. Available online: http://ec.europa.eu/ environment/eussd/smgp/product_footprint.htm (accessed on 28 September 2015).

7. European Commission Environmental Footprint Pilot Steering Committee and Technical Advisory Board Rules of Procedure. Available online: https://webgate.ec.europa.eu/fpfis/wikis/display/EUENVFP/ Steering+Committee+workspace?preview $=/ 63542841 / 101650704 /$ Rules $\% 20$ of $\% 20$ Procedure $\% 20 \% 20 \mathrm{EF} \%$ 20Steering\%20Committee\%20and\%20Technical\%20Advisory\%20Board.pdf (accessed on 15 January 2016).

8. European Commission Guidance for the Implementation of the EU Product Environmental Footprint (PEF) during the Environmental Footprint (EF) Pilot Phase-Version 5.0-August 2015. Available online: https:/ / webgate.ec.europa.eu/fpfis/wikis/download/attachments/80613270/Guidance_products_5.0_ final.doc?api=v2 (accessed on 6 January 2016).

9. Finkbeiner, M. Product environmental footprint-Breakthrough or breakdown for policy implementation of life cycle assessment? Int. J. Life Cycle Assess. 2014, 19, 266-271. [CrossRef]

10. Lehmann, A.; Bach, V.; Finkbeiner, M. Product Environmental Footprint in policy and market decisions-Applicability and impact assessment. Integr. Environ. Assess. Manag. 2015, 11, 417-424. [CrossRef] [PubMed]

11. ACEA. Joint Press Info: Environmental Footprint Methodology: Not for Us State Consumers and Manufactures. Available online: http://www.acea.be/press-releases/article/ joint_press_info_ environmental_footprint_methodology_not_for_us_state_consu (accessed on 15 January 2014).

12. BDI Position Paper. Design Product Environmental Footprint (PEF) in a Reasonable and Consistent Way! Available online: http://bdi.eu/media/themenfelder/umwelt/downloads/ umweltinformationen-produkte-und-dienstleistungen/Positionspapier_PEF_engl.pdf (accessed on 15 January 2016).

13. Eurometaux Open Letter to the PEF Pilot Project Steering Committee and Technical Advisory Board. May 2015. Available online: https:/ /webgate.ec.europa.eu/fpfis/wikis/download/attachments/63542841/2015 0511 open letter on PEF pilot exercise.pdf?api=v2 (accessed on 6 January 2016). 
14. BMUB/UBA/TUB Position Paper on EU Product and Organisation Environmental Footprint Proposal as Part of the Communication Building the Single Market for Green Products (COM/2013/0196 Final). Available online: https://webgate.ec.europa.eu/fpfis/wikis/download/attachments/63542841/Position paper on PEF_TUB_BMUB_UBA.pdf?api=v2 (accessed on 6 January 2016).

15. Galatola, M.; Pant, R. Reply to the editorial "Product environmental footprint-breakthrough or breakdown for policy implementation of life cycle assessment?" written by Prof. Finkbeiner (Int J Life Cycle Assess 19(2):266-271). Int J Life Cycle Assess 2014, 19, 1356-1360. [CrossRef]

16. Manfredi, S.; Allacker, K.; Pelletier, N.; Schau, E.; Chomkhamsri, K.; Pant, R.; Pennington, D. Comparing the European Commission product environmental footprint method with other environmental accounting methods. Int. J. Life Cycle Assess. 2015, 20, 389-404. [CrossRef]

17. European Commission Background Document for the Testing of Communication Vehicles in the Environmental Footprint Pilot Phase 2013-1016, Ver 1.1. Available online: https:/ /webgate.ec.europa.eu/ fpfis/wikis/download/attachments/66618509/Comm_bgdoc_v1.1.pdf?api=v2 (accessed on 6 January 2016).

18. Galatola, M.; Kerkhof, A.; Wouters, K. Determining the EF Benchmark and Performance Classes. Issue Paper. Version 2 (August 2015). Available online: https://webgate.ec.europa.eu/ fpfis/wikis/display/EUENVFP/Technical+Advisory+Board+workspace (accessed on 28 September 2015).

19. Gül, S.; Spielmann, M.; Lehmann, A.; Diana, E.; Bach, V.; Finkbeiner, M. Benchmarking and Environmental Performance Classes in Life Cycle Assessment-Development of a Procedure for Non-Leather Shoes in the Context of the Product Environmental Footprint. Int. J. Life Cycle Assess. 2015, 20, 1640-1648. [CrossRef]

20. European Commission. Regulation (EC) No 66/2010 of the European Parliament and of the Council of 25 November 2009 on the EU Ecolabel; European Commission: Brussels, Belgium; Luxembourg, Luxembourg, 2010.

21. European Union. Directive 2008/98/EC of the European Parliament and of the Council of 19 November 2008 on waste and repealing certain Directives. Available online: http://eur-lex.europa.eu/ legal-content/en/TXT/?uri=CELEX:32008L0098 (accessed on 6 January 2016).

22. European Commission-Joint Research Centre. International Reference Life Cycle Data System (ILCD) HandbookRecommendations for Life Cycle Impact Assessment in the European Context-Based on Existing Environmental Impact Assessment Models and f Actors; Publication Office of the European Union: Luxemburg, French, 2011.

23. Pfister, S.; Koehler, A.; Hellweg, S. Assessing the Environmental Impacts of Freshwater Consumption in LCA. Environ. Sci. Technol. 2009, 43, 4098-4104. [CrossRef] [PubMed]

24. Berger, M.; van der Ent, R.; Eisner, S.; Bach, V.; Finkbeiner, M. Water accounting and vulnerability evaluation (WAVE) - Considering atmospheric evaporation recycling and the risk of freshwater depletion in water footprinting. Environ. Sci. Technol. 2014, 48, 521-528. [CrossRef] [PubMed]

25. Schneider, L.; Berger, M.; Finkbeiner, M. Abiotic resource depletion in LCA-Background and update of the anthropogenic stock extended abiotic depletion potential (AADP) model. Int. J. Life Cycle Assess. 2015, 20, 709-721. [CrossRef]

26. Schneider, L.; Berger, M.; Finkbeiner, M. The anthropogenic stock extended abiotic depletion potential (AADP) as a new parameterisation to model the depletion of abiotic resources. Int. J. Life Cycle Assess. 2011, 16, 929-936. [CrossRef]

27. Finkbeiner, M.; Ackermann, R.; Bach, V.; Berger, M.; Brankatschk, G.; Chang, Y.-J.; Grinberg, M.; Lehmann, A.; Martínez-Blanco, J.; Minkov, N.; et al. Challenges in Life Cycle Assessment: An Overview of Current Gaps and Research Needs. In Background and Future Prospects in Life Cycle Assessment; Springer: Berlin, Germany; Heidelberg, Germany, 2014; pp. 207-258.

28. European Commission. Commission Regulation (EU) No 271/2010 of 24 March 2010 amending Regulation (EC) No 889/2008 laying down detailed rules for the implementation of Council Regulation (EC) No 834/2007, as regards the organic production logo of the European Union. Available online: http:/ / eur-lex.europa.eu/legal-content/EN/TXT/?uri=uriserv\%3AOJ.L_.2010.084.01.0019.01.ENG (accessed on 6 January 2016).

(C) 2016 by the authors; licensee MDPI, Basel, Switzerland. This article is an open access article distributed under the terms and conditions of the Creative Commons by Attribution (CC-BY) license (http:/ / creativecommons.org/licenses/by/4.0/). 